\title{
THE FIRST MASSIVE BLACK HOLE SEEDS AND THEIR HOSTS
}

\author{
Jillian Bellovary ${ }^{1}$, Marta Volonteri ${ }^{1}$, Fabio Governato $^{2}$, SiJing Shen $^{3}$, Thomas Quinn ${ }^{2}$, And James Wadsley ${ }^{4}$ \\ ${ }^{1}$ Department of Astronomy, University of Michigan, Ann Arbor, MI 48109, USA \\ ${ }^{2}$ Department of Astronomy, University of Washington, Seattle, WA 98195, USA \\ ${ }^{3}$ Department of Astronomy, University of California Santa Cruz, Santa Cruz, CA 95064, USA \\ ${ }^{4}$ Department of Physics and Astronomy, McMaster University, Hamilton, ON, Canada \\ Received 2011 April 19; accepted 2011 August 15; published 2011 November 2
}

\begin{abstract}
We investigate the formation of the first massive black holes (MBHs) in high redshift galaxies, with the goal of providing insights to which galaxies do or do not host MBHs. We adopt a novel approach to forming seed black holes in galaxy halos in cosmological $\mathrm{SPH}+\mathrm{N}$-body simulations. The formation of $\mathrm{MBH}$ seeds is dictated directly by the local gas density, temperature, and metallicity, and motivated by physical models of MBH formation. We explore seed black hole populations as a function of halo mass and redshift, and examine how varying the efficiency of MBH seed formation affects the relationship between black holes and their hosts. Seed black holes tend to form in halos with mass between $10^{7}$ and $10^{9} M_{\odot}$, and the formation rate is suppressed around $z=5$ due to the diffusion of metals throughout the intergalactic medium. We find that the time of MBH formation and the occupation fraction of black holes are a function of the host halo mass. By $z=5$, halos with mass $M_{\text {halo }}>3 \times 10^{9} M_{\odot}$ host MBHs regardless of the efficiency of seed formation, while the occupation fraction for smaller halos increases with black hole formation efficiency. Our simulations explain why MBHs are found in some bulgeless and dwarf galaxies, but we also predict that their occurrence becomes rarer and rarer in low-mass systems.
\end{abstract}

Key words: black hole physics - galaxies: evolution - galaxies: formation - galaxies: high-redshift - methods: numerical

Online-only material: color figures

\section{INTRODUCTION}

Massive black holes (MBHs) are commonly found in massive galaxies with a significant bulge component (Gehren et al. 1984), but recent discoveries show that they can also be found in bulgeless disk galaxies (Filippenko \& Ho 2003; Shields et al. 2008) and dwarfs (Barth et al. 2004; Reines et al. 2011). It is then unclear whether bulges are related to MBH formation processes, what fraction of low-mass galaxies may host MBHs, and whether MBHs in these galaxies have an important role in galaxy evolution. In this paper, we present a novel approach to seed MBH formation based on the properties of metal-free gas which explains the existence of MBHs in dwarf galaxies (e.g., Reines et al. 2011). Our simulations, however, suggest that $\mathrm{MBHs}$ become infrequent in low-mass galaxies, and that a minimum galaxy mass exists below which MBHs become progressively uncommon.

MBHs must have originated from moderately massive "seed" black holes in order to grow to a billion solar masses by $z \simeq 6$ (Haiman \& Loeb 2001). These seeds must form at high redshifts $(z \sim 15-30)$ and grow rapidly in order to reproduce the observed distribution of high redshift quasars (Volonteri et al. 2003). Seeds may be the remnants of Population III stars, which form with extremely low metallicity and thus have unique properties. It is commonly speculated that Population III stars have a topheavy initial mass function (IMF), with masses ranging from 100 to $1000 M_{\odot}$ (e.g., Couchman \& Rees 1986; Abel et al. 2002; Bromm \& Larson 2004). Any zero-metallicity star with a mass greater than $\sim 260 M_{\odot}$ will leave a $\sim 100 M_{\odot}$ black hole behind (Bond et al. 1984; Heger \& Woosley 2002).

Another theory involves the direct collapse of very metalpoor, low-angular momentum gas via dynamical instabilities (Oh \& Haiman 2002; Loeb \& Rasio 1994; Eisenstein \& Loeb 1995; Koushiappas et al. 2004; Begelman et al. 2006; Lodato
\& Natarajan 2006). If enough gas is funneled into the center of a local overdensity, it may collapse to form a black hole with mass $10^{4}-10^{6} M_{\odot}$ (Begelman et al. 2006; Lodato \& Natarajan 2006; Begelman et al. 2008). This process may happen later than Population III star formation, because halos must be larger to host such massive inflow. Efficient gas collapse is more likely to occur in massive halos with virial temperatures $T_{\text {vir }}>10^{4} \mathrm{~K}$ under metal-free conditions where the formation of $\mathrm{H}_{2}$ is inhibited by a UV background (Bromm \& Loeb 2003) and cooling is dominated by atomic hydrogen. In such halos, fragmentation is suppressed, cooling proceeds gradually, and the gaseous component can cool and participate in MBH formation before it is turned into stars. These halos may need to exist in regions of ultracritical UV radiation in order to form MBHs by direct collapse (Dijkstra et al. 2008; Shang et al. 2010), since the average estimated UV background may not be sufficient to prevent some Population III stars from forming in halos of this size (Johnson et al. 2008).

While the processes which may lead to MBH seed formation have been modeled cosmologically (Wise et al. 2008; Regan \& Haehnelt 2009), it is not yet possible to do so in simulations involving volumes larger than a few $\mathrm{Mpc}^{3}$. Previous large-scale simulations have incorporated seed black hole formation in a simplistic way. Sijacki et al. (2007), Di Matteo et al. (2008), and Booth \& Schaye (2009) all employ similar methods, which involves running an on-the-fly halo finder on the simulation as it evolves, and planting seed MBHs in particular halos by hand. The halos are chosen based on a mass threshold (generally $M_{\text {halo }} \sim 10^{10} M_{\odot}$ ), and seeds are planted if there is not already a black hole present. There is no metallicity criterion, so seed MBHs can form at any redshift if a halo meets the eligibility criteria. The seeds are placed at the center of the halo and generally fixed there throughout the remainder of the simulation (Booth \& Schaye 2009). 
Table 1

Simulation Properties

\begin{tabular}{|c|c|c|c|c|c|c|c|}
\hline Simulation & $\begin{array}{c}\text { Halo Mass } \\
\text { at } z=5\left(M_{\odot}\right)\end{array}$ & $\begin{array}{l}\text { Gas Particle } \\
\text { Mass }\left(M_{\odot}\right)\end{array}$ & $\begin{array}{l}\text { Gas/DM } \\
\text { Ratio }\end{array}$ & $\begin{array}{l}\text { Softening } \\
\quad(\mathrm{kpc})\end{array}$ & $\chi_{\text {seed }}$ & $\begin{array}{c}\text { No. of BHs } \\
\text { at } z=5\end{array}$ & $\begin{array}{c}n_{\min } \\
\left(\operatorname{amu~cm}{ }^{-3}\right)\end{array}$ \\
\hline h603 & $8.48 \times 10^{9}$ & 26676 & 1.69 & 0.173 & $0.05,0.1,0.3,0.5$ & $33,44,51,49$ & 10 \\
\hline h258 & $2.92 \times 10^{10}$ & 26676 & 0.713 & 0.173 & $0.05,0.1,0.3,0.5$ & $39,56,76,84$ & 10 \\
\hline hz1 & $5.88 \times 10^{11}$ & 90031 & 0.713 & 0.260 & $0.05,0.1,0.3,0.5$ & $166,240,465,504$ & 2.5 \\
\hline
\end{tabular}

However, there is no physical motivation for a halo mass threshold for $\mathrm{MBH}$ placement of $10^{10} M_{\odot}$, and in fact seeds likely form in halos of much lower mass (see Section 3.2). This method also prevents more than one seed from forming per halo, which may be unrealistic-halos may experience the formation of multiple Population III stars if fragmentation occurs (Turk et al. 2009; Stacy et al. 2010; Greif et al. 2011; Clark et al. 2011). Fixing the black hole at the halo center is similarly inadvisable-dynamical effects such as galaxy mergers or gravitational recoil may cause a black hole to temporarily vacate the exact center of its galaxy, but such a circumstance is prohibited in these models.

The formalism of seed $\mathrm{MBH}$ formation we describe in this paper is a more sophisticated and realistic model because it makes no assumptions about the underlying halo properties. Our method relies only on the prospective MBH's local environment to determine when and where the $\mathrm{MBH}$ forms, including a requirement for zero-metallicity gas. Our scenario is broadly consistent with either proposed $\mathrm{MBH}$ formation mechanism, and also allows MBHs to evolve dynamically in a realistic way. It is crucial to study how variations in halo mass affect the frequency of formation when examining simulated seed $\mathrm{MBH}$ populations, and so we include in our sample a variety of halo masses in order to present a more coherent picture of high- $z$ seed formation and evolution as a function of cosmic environment. With this technique, we investigate where and when MBH seeds form at high redshift, and discuss the implications for galaxies at $z=0$.

\section{THE SIMULATIONS}

We employ the SPH $+N$-Body Tree code Gasoline (Stadel 2001; Wadsley et al. 2004), which has been shown to produce realistic galaxies in cosmological simulations (e.g., Governato et al. 2010; Stinson et al. 2010; Pontzen et al. 2010; Oh et al. 2011; Brooks et al. 2011). Gasoline includes a physically motivated prescription for star formation as well as a recipe for the formation and evolution of MBH seeds (described in Section 3). We use a Kroupa IMF (Kroupa 2001) and a WMAP3 cosmology (Spergel et al. 2007). We use a supernova feedback model which incorporates the Sedov solution to the blast wave equations (see Stinson et al. 2006) and set the blast wave energy to $E_{\mathrm{SN}}=10^{51} \mathrm{erg}$. We implement a uniform ionizing radiation background with an onset at redshift $z=9$ (Haardt \& Madau 1996). Our simulations include cooling through metal lines as well as a model for turbulent metal diffusion (Shen et al. 2010); however, we do not including cooling via molecular hydrogen in the simulations presented here.

To probe a large parameter space, we employ a suite of simulations with different halo masses to test seed $\mathrm{MBH}$ formation. It is crucial to study how variations in halo mass affect the frequency of formation when examining simulated seed $\mathrm{MBH}$ populations. We have chosen a set of three fiducial halos selected from a uniform resolution, $50 \mathrm{Mpc}$ volume and resimulated at high resolution using the volume renormalization technique (Katz \& White 1993). We sample the region of interest at high resolution, and then sample more coarsely as the distance from the chosen object increases. This technique results in a large dynamic range, where we can capture the detailed physics of galaxy formation on small scales in the region of interest, and also the large-scale tidal torques from structures several Mpc away. The three chosen halos correspond broadly to a low-mass disk galaxy (h603), a Milky Way-mass disk galaxy (h258), and a massive elliptical galaxy ( $h z 1)$ at $z=0$. We present the details of our simulations in Table 1. Our simulations are extremely high resolution, with gas particles having masses between $2-9 \times 10^{4}$ $M_{\odot}$, and a force resultion of $173-260 \mathrm{pc}$, depending on the simulation. The mass ratio of gas and dark matter particles (Column 4) is of order unity, which reduces the effects due to two-body scattering and is critical for keeping $\mathrm{MBHs}$ in the centers of their galaxies.

Since we are interested in the epoch of seed $\mathrm{MBH}$ formation, we have run our simulations to $z=5$ rather than $z=0$, in order to maximize resolution while using only modest computational resources. We use the Amiga Halo Finder (Gill et al. 2004; Knollmann \& Knebe 2009) to identify galaxy halos based on the overdensity criterion for a flat universe (Gross 1997). For each simulated region, we analyze $\mathrm{MBH}$ populations for the primary halo as well as every satellite with at least 64 particles. In the case of $h 603$ there are 5370 total halos in our analysis; for $h 258$ there are 5170; for $h z 1$ there are 2160.

\section{SEED FORMATION AND EVOLUTION}

Since the actual physical process of $\mathrm{MBH}$ seed formation is unresolvable in cosmological simulations, we have developed a model which is broadly consistent with both of the proposed seed formation scenarios. The common thread between the proposed scenarios is an ability for gas to collapse into a large central mass, which requires a zero or near-zero metallicity (though see Mayer et al. 2010 for an alternate mechanism). Because our star formation prescription is already based on similar physics (i.e., cold, dense, collapsing gas results in the formation of star particles), we use this prescription with the additional criterion of zero metallicity to form seed MBHs.

\subsection{MBH Formation}

Our current star formation recipe is described in detail in Stinson et al. (2006), and we summarize it here. For a star to form in our simulations, its parent gas particle must meet several criteria. Primarily, the gas density must be greater than the threshold density for star formation, $n_{\min }$. In simulations with force resolution of $\lesssim 100 \mathrm{pc}$, high density peaks can be resolved, mimicking star formation regions in giant molecular clouds (Governato et al. 2010). In this instance, one can use a realistic value of $n_{\min }=100 \mathrm{amu} \mathrm{cm}{ }^{-3}$. However, at lower resolution we cannot properly resolve these high density peaks, and in order to match observed relations such as the Kennicutt-Schmidt 

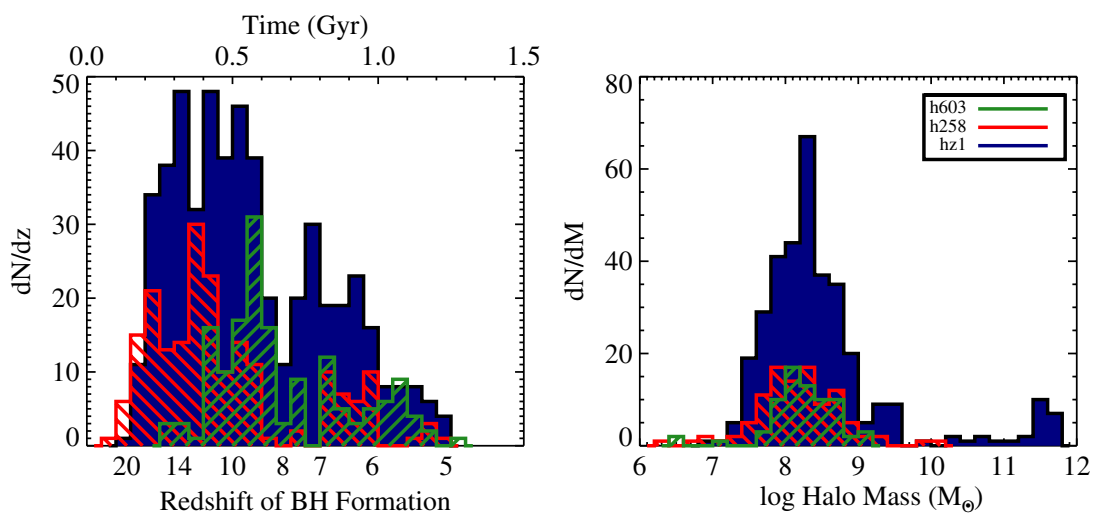

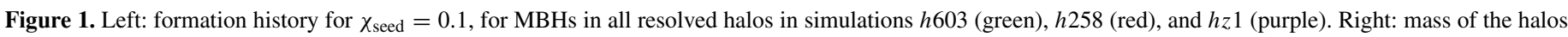
at the time the $\mathrm{MBH}$ formed for $\chi_{\text {seed }}=0.1$.

(A color version of this figure is available in the online journal.)

law (Kennicutt 1989) and the Tully-Fisher relation, we use a lower value (related to the mass and spatial resolution) for $n_{\text {min }}\left(2.5-10 \mathrm{amu} \mathrm{cm}{ }^{-3}\right)$. These values are presented in Table 1 for each simulation in our study. In addition to the density criterion, the gas temperature $T$ must be less than a fiducial temperature $T_{\max }$, which we set to $1.5 \times 10^{4} \mathrm{~K}$. Star formation efficiency is governed by the free parameter, $c^{*}$, which we set to 0.1 (Governato et al. 2010) to match the observed relations mentioned above. If a gas particle meets all of the relevant criteria, the probability it will form a star is given by

$$
p=\frac{m_{\mathrm{gas}}}{m_{\mathrm{star}}}\left(1-e^{c^{*} \Delta t / t_{\mathrm{form}}}\right),
$$

where $m_{\text {star }}$ and $m_{\text {gas }}$ are the star and gas particle masses, $t_{\text {form }}$ is the dynamical time for the gas particle, and $\Delta t$ is the time between star formation episodes, which we set to $1 \mathrm{Myr}$.

For a seed $\mathrm{MBH}$ to form, all of the criteria for star formation must be met in addition to the criterion of zero metallicity. If a gas particle meets the criteria to form a star and probabilistically is able to do so, it then has an additional probability to instead form an $\mathrm{MBH}$. The probability to form an $\mathrm{MBH}$ seed is given by the above expression multiplied by the newly introduced parameter $\chi_{\text {seed }}$, which sets the approximate number of seed MBHs which will form in a given simulation. In this paper, we investigate how varying this parameter affects $\mathrm{MBH}$ populations in high-redshift galaxies with a range of masses, and explore values of $\chi_{\text {seed }}$ between 0.05 and 0.5 . This parameter range is motivated by models predicting the efficiency of seed $\mathrm{MBH}$ progenitors. For example, according to Lodato \& Natarajan (2006) the distribution of halos which might host direct-collapse MBHs varies between $4 \%$ and $35 \%$ for a reasonable range of halo spins, virial temperatures, and the Toomre parameter $Q$. Additionally, the Population III star IMF reported by Tan et al. (2010) has a mean mass of $250 M_{\odot}$, which is roughly the mass where one expects an $\mathrm{MBH}$ remnant to form; therefore $\sim 50 \%$ of Population III stars may form MBH seeds. Thus we believe our $\chi_{\text {seed }}$ range of $0.05-0.5$ samples the parameter space of current theoretical predictions.

When seed MBHs do form, they acquire the mass of their parent gas particle. Due to resolution limitations, we cannot form MBH seeds with Population III remnant masses $\left(\sim 100 M_{\odot}\right)$, but one can imagine a scenario in which Pop III seeds form at high $z$ and grow through mergers and accretion to the higher masses we employ in our simulations (Li et al. 2007). Our seed mass is therefore broadly consistent with either seed formation scenario. MBHs are allowed to merge if they become close together in space (within two softening lengths) and have low relative velocities. Specifically, they must fulfill the criterion $\frac{1}{2} \Delta \vec{v}^{2}<\Delta \vec{a} \cdot \Delta \vec{r}$, where $\Delta \vec{v}$ and $\Delta \vec{a}$ are the differences in velocity and acceleration of the two black holes, and $\Delta \vec{r}$ is the distance between them. In this study, we do not enable MBHs to grow through gas accretion, nor do we include any type of feedback from MBHs. We have chosen to omit these aspects of $\mathrm{MBH}$ physics to gain a robust lower limit on $\mathrm{MBH}$ seed populations. We verified that accretion and feedback do not affect our results by performing a simulation of $h 258$ with the inclusion of these processes (assuming Bondi-Hoyle accretion and a feedback efficiency of 1\%; see Bellovary et al. 2010 for more details), and found that there were no significant differences in $\mathrm{MBH}$ growth or star formation history with their inclusion. At the high redshifts we are probing here, such $\mathrm{MBH}$ activity is only efficient in the largest halos, which are rare in the majority of the simulations we present in this work.

\subsection{MBH Formation History}

A detailed understanding of the formation history of $\mathrm{MBHs}$, as well as the host halo mass at the time of $\mathrm{MBH}$ formation, is essential for interpreting our results. In Figure 1 (left panel), we show the formation history of seed MBHs for every resolved halo in each galaxy for $\chi_{\text {seed }}=0.1$. Changing $\chi_{\text {seed }}$ does not qualitatively change the $\mathrm{MBH}$ formation history, it simply results in a different number of $\mathrm{MBH}$ seeds formed (higher values of $\chi_{\text {seed }}$ result in increased seed formation and consequently an increased MBH-MBH merger rate-see Table 1 for the number of MBHs in each simulation at $z=5$ ). Comparing simulations $h 603$ (green histogram) and $h 258$ (red histogram), we see the clear trend of an earlier onset of $\mathrm{MBH}$ formation with larger overdensities. The peaks in $\mathrm{MBH}$ formation are offset by 200-400 Myr for simulations $h 603$ and $h 258$ due to this effect of cosmic bias (Peebles 1980). This effect is not evident between simulations $h 258$ and $h z 1$ (purple histogram) due to the stochastic nature of cosmic large-scale structure. However, though simulations $h z 1$ and $h 258$ begin forming stars and MBHs at the same time, these objects form at a much more rapid rate in $h z 1$ due to the overdense nature of the selected region. The $\operatorname{dip}$ at $z=9$ for each simulation is a result of the onset of the UV background at that time, when a substantial amount of cool gas is heated above $T_{\max }$. In all galaxies the rate of seed formation dwindles around $z=5$, because the majority of halos have experienced supernova explosions and have been metal- 


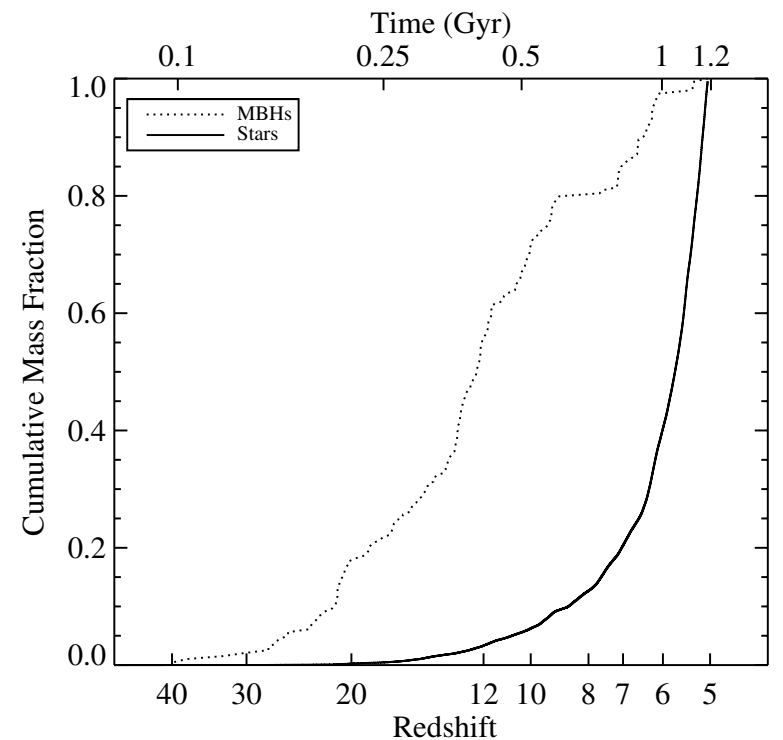

Figure 2. Cumulative mass fraction for stars (solid line) and MBHs (dotted line) for simulation $h 258$ with $\chi_{\text {seed }}=0.1$.

enriched, which in turn quenches seed MBH formation. At this point, MBH seeds can only form in areas which have not yet experienced local star formation, which are generally restricted to the outskirts of the simulation.

The connection between star formation, metal pollution, and $\mathrm{MBH}$ formation is exemplified in Figure 2. Here, we show the cumulative mass fraction of stars and MBHs for all of the galaxies in simulation $h 258\left(\chi_{\text {seed }}=0.1\right)$. The bulk of the MBHs form while the star formation rate is still quite low. $\mathrm{MBH}$ formation tapers off at $z=5$, at a time when the star formation rate is increasing rapidly. These effects are due to the metallicity criterion for seed formation; MBHs form efficiently only until metals have diffused through the interstellar gas, at which point $\mathrm{MBH}$ formation is truncated fairly quickly.

We next examine the mass of a halo at the time a black hole seed forms within it. Figure 1 (right panel) shows that in our simulations seed MBHs form in halos which are less massive than the $M_{\text {halo }}=10^{10} M_{\odot}$ assumed in previous works. Our predicted halo mass range is between $10^{7}$ and $10^{9} M_{\odot}$, with a peak around $10^{8} M_{\odot}$, and does not vary with $\chi_{\text {seed }}$. (Again we show only $\chi_{\text {seed }}=0.1$, the other values of $\chi_{\text {seed }}$ give nearly identical results.) This halo mass is consistent with that in which Population III stars are expected to form $\left(>10^{6}\right.$ $M_{\odot}$; Couchman \& Rees 1986; Barkana \& Loeb 2001), and also with the halo mass expected for seed $\mathrm{MBH}$ formation via direct collapse scenario $\left(\sim 10^{7} M_{\odot}\right.$, Koushiappas et al. 2004; Begelman et al. 2006; Lodato \& Natarajan 2006). This agreement is simply a consequence of the physics of gas cooling in virialized halos; though we do not include prescriptions for molecular hydrogen cooling or Population III star formation, we find good agreement with predictions of the first collapsing metal-free gas clouds. The halo mass at time of $\mathrm{MBH}$ formation is slightly dependent on the resolution of the simulation in question, in that the distribution shifts toward smaller masses with higher resolution, but this effect is minimal and we do not expect our results to differ strongly if we move to yet higher resolution. Also note that because more than one $\mathrm{MBH}$ is allowed to form in a halo, a particular halo may be represented more than once in the right panel of Figure 1.
The halo mass at the time of $\mathrm{MBH}$ formation is somewhat dependent on the cosmic overdensity, in that $\mathrm{MBH}$ formation (and star formation in general) begins earlier in volumes with larger density perturbations. This phenomenon leads to the earliest onsets of star formation occurring in halos which are slightly smaller than those in less overdense regions. For the galaxy $h z 1$, the distribution of halos is actually bimodal, with a strong peak around $M_{\text {halo }} \sim 10^{8} M_{\odot}$, and a secondary peak at large halo mass. The MBHs which form in large halos do so at late times (after the primary burst of $\mathrm{MBH}$ formation) in the outskirts of the primary halo (between 20 and 50 physical kpc from the center) in pockets of very low metallicity. These $\mathrm{MBH}$ formation events may be a resolution effect, as they may be occurring in pressure-confined clouds as reported in Kaufmann et al. (2006); with higher resolution (and a corresponding higher density threshold for star/MBH formation) these objects may cease to exist. We plan to explore this effect further with high resolution tests. However, since these objects form in low density regions, they will exist as "wandering" $\mathrm{MBHs}$ in the halo and never inspiral to the center to merge with the primary.

Allowing MBH seeds to form in halos with $M_{\text {halo }}<10^{10} M_{\odot}$ is vital for capturing their full evolution. While several of these seeds will eventually become central MBHs in large halos at $z=0$, many may have other fates. For example, when small halos hosting $\mathrm{MBH}$ seeds merge with larger ones, they may undergo tidal stripping, resulting in a "wandering" black hole in the larger galaxy halo (Bellovary et al. 2010). If the satellite halo is not tidally destroyed, it still may have been stripped of enough baryons to quench the growth of its $\mathrm{MBH}$, resulting in a dwarf galaxy hosting an intermediate-mass black hole which may be near its initial seed mass.

\section{MBH-HALO OCCUPATION FRACTION}

The frequency at which MBHs occupy halos is a fundamental measure of MBH seed formation efficiency. There are constraints on this quantity in the local universe, albeit weak ones; for example, nuclear activity due to $\mathrm{MBH}$ sas been detected in $32 \%$ of the late-type galaxies in the Virgo Cluster, exclusively in galaxies with mass $M_{\text {halo }}>10^{10} M_{\odot}$ (Decarli et al. 2007). For early-type galaxies in Virgo, nuclear activity exists in 3\%-44\% of galaxies with mass less than $10^{10} M_{\odot}$ and 49\%-87\% of galaxies with mass greater than $10^{10} M_{\odot}$ (Gallo et al. 2008). Such estimates put a lower limit on the $\mathrm{MBH}$ occupation fraction of galaxies in this cluster, since inactive black holes are unlikely to be observed. Observations at higher redshifts are more challenging, though data at $z \sim 1$ from DEEP2 and AEGIS may help provide some constraints (see Yan et al. 2011), and the synergy of the James Webb Space Telescope (JWST) and Atacama Large Millimeter Array (ALMA) will more robustly probe occupation fractions in distant galaxies.

Theoretical constraints of the MBH occupation fraction are also quite weak. Semi-analytic models show that the occupation fraction varies depending on which method of $\mathrm{MBH}$ formation is used (van Wassenhove et al. 2010) or how efficiently seeds are formed (Volonteri et al. 2008b; Tanaka \& Haiman 2009). Only a few percent of high-redshift halos need host MBHs in order to reproduce the $z=0$ occupation fraction (Menou et al. 2001). However, high-redshift occupation fractions anywhere from $10 \%$ to $100 \%$ can reproduce the observed quasar luminosity function and supermassive black hole (SMBH) mass function, depending on parameters such as radiative efficiency and quasar duty cycle (Lippai et al. 2009). Clearly much uncertainty remains regarding the high-redshift $\mathrm{MBH}$ occupation fraction, 


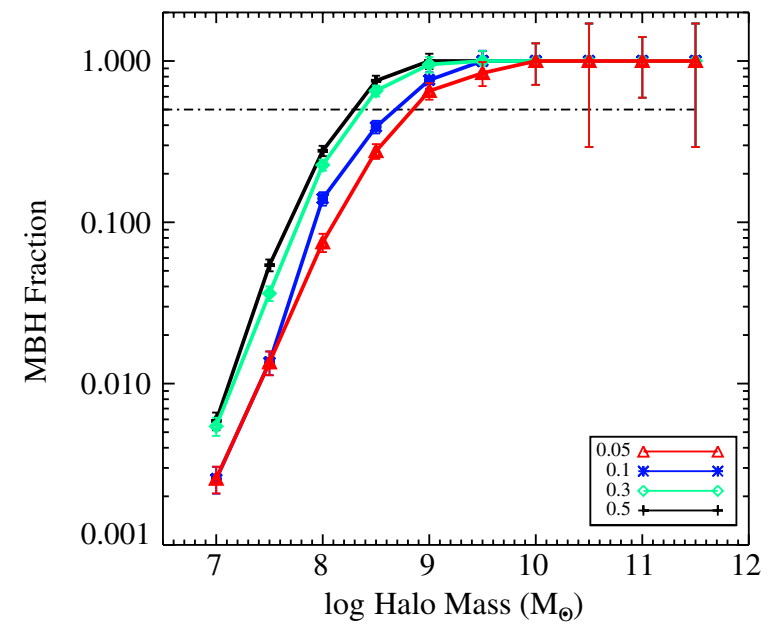

Figure 3. $\mathrm{MBH}$-halo occupation fraction for a given halo mass, for the parent galaxy and subhalos of all three simulations at $z=5$. Colored lines and symbols represent simulations with different values of $\chi_{\text {seed }}$ (red triangles: $\chi_{\text {seed }}=0.05$, blue asterisks: $\chi_{\text {seed }}=0.1$, green diamonds: $\chi_{\text {seed }}=0.3$, and black crosses: $\chi_{\text {seed }}=0.5$ ). Error bars represent uncertainty due to Poisson statistics. The horizontal dot-dashed line marks where the probability that a galaxy hosts an $\mathrm{MBH}$ is $50 \%$, corresponding to halo masses between $10^{8}$ and $10^{9} \mathrm{M}_{\odot}$.

(A color version of this figure is available in the online journal.)

and we take this opportunity to explore how halo mass and seed formation efficiency affect this quantity.

In Figure 3, we show the MBH-halo occupation fraction versus halo mass for the four values of $\chi_{\text {seed }}$. Here, we include the parent galaxy and subhalos of all three simulations together at $z=5$. Halos with virial masses above $\log \left(M_{\text {halo }}\right) \sim 9.5$ always host an MBH seed, regardless of the value of seed formation efficiency. Even in the lowest efficiency case, $\mathrm{MBH}$ seeds form in the regions of earliest star formation, which tend to be the halos which become the most massive later on in every simulation. The most massive halos have also experienced the greatest number of mergers, which further populates them with $\mathrm{MBH}$ seeds brought in by satellites. Thus, our model predicts that halos with masses greater than $M_{\text {halo }} \sim 10^{9} M_{\odot}$ will be extremely likely to host $\mathrm{MBH}$ seeds, even if the formation efficiency of such seeds is small. This result is consistent with observations of the local universe, where MBHs are found to occupy halos above a similar mass threshold with high likelihood (Ferrarese et al. 2006; Wehner \& Harris 2006).

To estimate the fate of MBHs at later cosmic times, we have run a lower-resolution simulation of galaxy $h 258$ to $z=0$ and traced the mass evolution of halos. Halos with mass between $10^{7} M_{\odot}$ and $10^{10} M_{\odot}$ at $z=5$ can remain in the same mass range at $z=0$, be stripped, or become incorporated in the main halo. In this particular case, $\sim 30 \%$ of halos with $M_{\text {halo }}>10^{9}$ $M_{\odot}$ - that host MBHs already at $z=5$-are stripped during their evolution, ending up as halos with $z=0$ masses of $10^{8}-10^{10} M_{\odot}$. While one zoomed-in simulation cannot give us broad statistics on the evolution of all $z=5$ halos, we can deduce that while a large number of high-redshift $\mathrm{MBH}$ hosts undergo hierarchical merging and settle in massive galaxies, a non-negligible fraction have more quiescent merger histories and do not grow substantially, and make up today's population of low-mass galaxies which may harbor MBHs.

On the other hand, the occupation fraction of halos with masses less than $M_{\text {halo }} \sim 10^{9} M_{\odot}$ is sensitive to the physics of $\mathrm{MBH}$ formation, via $\chi_{\text {seed }}$. For example, in galaxies $h 258$ and $h z 1$, halos with mass $M \sim 10^{9} M_{\odot}$ are nearly $100 \%$ likely to host an MBH seed if $\chi_{\text {seed }}=0.5$, but only $10 \%$ likely when $\chi_{\text {seed }}=0.05$. The dependence of occupation fraction with $\chi_{\text {seed }}$ becomes weaker in galaxy $h 603$, however. We suggest that in the larger halos, metals diffuse throughout the simulation more efficiently (due to increased star formation and possible ejection of metals from galaxies) which suppresses $\mathrm{MBH}$ formation in their satellites. In the isolated low-mass disk galaxy, however, the relative lack of metal diffusion allows MBHs to continue forming in these halos at a time when $\mathrm{MBH}$ formation would have been truncated in a larger overdensity. In this study we have aimed for high resolution with a sample of field galaxies; for a simulated uniform volume, including voids and high density regions (which more completely samples halos in the universe) our results may be slightly different. However, a test study using a small $(6 \mathrm{Mpc})$ uniform volume gives results consistent with our findings here.

\section{SUMMARY}

We have undertaken a study of the formation and evolution of seed MBHs using cosmological simulations, including a unique and physically motivated recipe for seed $\mathrm{MBH}$ formation. In our simulations, $\mathrm{MBH}$ formation depends solely on the local properties of the surrounding gas (i.e., density, temperature, metallicity) rather than on global halo properties. We can thus model the evolution of MBH seeds within their halos in a cosmological framework in a fully self-consistent way.

We find that MBHs form in a burst during the onset of the earliest star formation, but the formation rate tapers off by $z=5$ due to the diffusion of metals throughout the intergalactic medium. $\mathrm{MBH}$ formation is concentrated in halos with masses between $10^{7}$ and $10^{9} M_{\odot}$, consistent with predictions of seed SMBH formation (Couchman \& Rees 1986; Barkana \& Loeb 2001; Koushiappas et al. 2004; Begelman et al. 2006; Lodato \& Natarajan 2006). MBHs which exist in small halos at high redshift may contribute to the $z=0$ population of "wandering" black holes in massive galaxies, or they may appear as intermediate-mass black holes in low mass and/or bulgeless galaxies (Greene \& Ho 2007) if their hosts have quiescent merger histories.

We find that the time of black hole formation and the occupation fraction of black holes are a function of the host halo mass. Large halos form MBHs earlier and they are more likely to host an $\mathrm{MBH}$. An observational determination the $\mathrm{MBH}$-halo occupation fraction for halos of mass $M_{\text {halo }}<10^{9} M_{\odot}$ would be a strong constraint on the true formation efficiency of $\mathrm{MBH}$ seeds (see also Volonteri et al. 2008a, 2008b; van Wassenhove et al. 2010). In a forthcoming paper, we will investigate the coevolution of MBHs and galaxies at later cosmic times, as well as derive both the occupation fraction and the active fraction of MBHs in galaxies. The latter is a direct observational constraint; a preliminary study of the active fraction at $z=1$ is already possible now with data from DEEP2 and AEGIS, while local high sensitivity studies are being carried for both field galaxies (B. Miller et al. 2011, in preparation) and in the Virgo Cluster (Gallo et al. 2010).

It may be possible that some MBHs could form at even lower redshifts in halos near cosmic voids. Since these areas have below-average densities, they will have experienced far less star formation and thus will have a lower metallicity than their high-density counterparts. Such events have been predicted for Population III stars at redshifts from $2<z<6$ (Jimenez \& Haiman 2006; Tornatore et al. 2007; Trenti et al. 2009). Searches for seed MBH formation in high-redshift void galaxies 
may provide unique observational clues regarding the origins of supermassive black holes.

Simulations were run using computer resources and technical support from NAS. M.V. acknowledges support from SAO Award TM1-12007X and NASA awards ATP NNX10AC84G and NNX07AH22G. F.G. acknowledges support from an NSF grant AST-0607819 and NASA ATP NNX08AG84G. J.B. and T.Q. acknowledge support from NASA Grant NNX07AH03G. The authors also thank Kayhan Gültekin, Brendan Miller, Michele Trenti, and the anonymous referee for providing insights which improved the paper.

\section{REFERENCES}

Abel, T., Bryan, G. L., \& Norman, M. L. 2002, Science, 295, 93

Barkana, R., \& Loeb, A. 2001, Phys. Rep., 349, 125

Barth, A. J., Ho, L. C., Rutledge, R. E., \& Sargent, W. L. W. 2004, ApJ, 607, 90

Begelman, M. C., Rossi, E. M., \& Armitage, P. J. 2008, MNRAS, 387, 1649

Begelman, M. C., Volonteri, M., \& Rees, M. J. 2006, MNRAS, 370, 289

Bellovary, J. M., Governato, F., Quinn, T. R., et al. 2010, ApJ, 721, L148

Bond, J. R., Arnett, W. D., \& Carr, B. J. 1984, ApJ, 280, 825

Booth, C. M., \& Schaye, J. 2009, MNRAS, 398, 53

Bromm, V., \& Larson, R. B. 2004, ARA\&A, 42, 79

Bromm, V., \& Loeb, A. 2003, ApJ, 596, 34

Brooks, A. M., Solomon, A. R., Governato, F., et al. 2011, ApJ, 728, 51

Clark, P. C., Glover, S. C. O., Klessen, R. S., \& Bromm, V. 2011, ApJ, 727, 110

Couchman, H. M. P., \& Rees, M. J. 1986, MNRAS, 221, 53

Decarli, R., Gavazzi, G., Arosio, I., et al. 2007, MNRAS, 381, 136

Di Matteo, T., Colberg, J., Springel, V., Hernquist, L., \& Sijacki, D. 2008, ApJ, 676,33

Dijkstra, M., Haiman, Z., Mesinger, A., \& Wyithe, J. S. B. 2008, MNRAS, 391, 1961

Eisenstein, D. J., \& Loeb, A. 1995, ApJ, 443, 11

Ferrarese, L., Côté, P., Jordán, A., et al. 2006, ApJS, 164, 334

Filippenko, A. V., \& Ho, L. C. 2003, ApJ, 588, L13

Gallo, E., Treu, T., Jacob, J., et al. 2008, ApJ, 680, 154

Gallo, E., Treu, T., Marshall, P. J., et al. 2010, ApJ, 714, 25

Gehren, T., Fried, J., Wehinger, P. A., \& Wyckoff, S. 1984, ApJ, 278, 11

Gill, S. P. D., Knebe, A., \& Gibson, B. K. 2004, MNRAS, 351, 399

Governato, F., Brook, C., Mayer, L., et al. 2010, Nature, 463, 203

Greene, J. E., \& Ho, L. C. 2007, ApJ, 670, 92

Greif, T. H., Springel, V., White, S., et al. 2011, ApJ, 737, 75

Gross, M. A. K. 1997, PhD thesis, Univ. California, Santa Cruz

Haardt, F., \& Madau, P. 1996, ApJ, 461, 20

Haiman, Z., \& Loeb, A. 2001, ApJ, 552, 459
Heger, A., \& Woosley, S. E. 2002, ApJ, 567, 532

Jimenez, R., \& Haiman, Z. 2006, Nature, 440, 501

Johnson, J. L., Greif, T. H., \& Bromm, V. 2008, MNRAS, 388, 26

Katz, N., \& White, S. D. M. 1993, ApJ, 412, 455

Kaufmann, T., Mayer, L., Wadsley, J., Stadel, J., \& Moore, B. 2006, MNRAS, 370,1612

Kennicutt, R. C., Jr. 1989, ApJ, 344, 685

Knollmann, S. R., \& Knebe, A. 2009, ApJS, 182, 608

Koushiappas, S. M., Bullock, J. S., \& Dekel, A. 2004, MNRAS, 354, 292

Kroupa, P. 2001, MNRAS, 322, 231

Li, Y., Hernquist, L., Robertson, B., et al. 2007, ApJ, 665, 187

Lippai, Z., Frei, Z., \& Haiman, Z. 2009, ApJ, 701, 360

Lodato, G., \& Natarajan, P. 2006, MNRAS, 371, 1813

Loeb, A., \& Rasio, F. A. 1994, ApJ, 432, 52

Mayer, L., Kazantzidis, S., Escala, A., \& Callegari, S. 2010, Nature, 466, 1082

Menou, K., Haiman, Z., \& Narayanan, V. K. 2001, ApJ, 558, 535

Oh, S., Brook, C., Governato, F., et al. 2011, AJ, 142, 24

Oh, S. P., \& Haiman, Z. 2002, ApJ, 569, 558

Peebles, P. J. E. (ed.) 1980, The Large-scale Structure of the Universe (Princeton, NJ: Princeton Univ. Press)

Pontzen, A., Deason, A., Governato, F., et al. 2010, MNRAS, 402, 1523

Regan, J. A., \& Haehnelt, M. G. 2009, MNRAS, 396, 343

Reines, A. E., Sivakoff, G. R., Johnson, K. E., \& Brogan, C. L. 2011, Nature, 470, 66

Shang, C., Bryan, G. L., \& Haiman, Z. 2010, MNRAS, 402, 1249

Shen, S., Wadsley, J., \& Stinson, G. 2010, MNRAS, 407, 1581

Shields, J. C., Walcher, C. J., Böker, T., et al. 2008, ApJ, 682, 104

Sijacki, D., Springel, V., di Matteo, T., \& Hernquist, L. 2007, MNRAS, 380, 877

Spergel, D. N., Bean, R., Doré, O., et al. 2007, ApJS, 170, 377

Stacy, A., Greif, T. H., \& Bromm, V. 2010, MNRAS, 403, 45

Stadel, J. G. 2001, PhD thesis, AA (Univ. Washington)

Stinson, G., Seth, A., Katz, N., et al. 2006, MNRAS, 373, 1074

Stinson, G. S., Bailin, J., Couchman, H., et al. 2010, MNRAS, 408, 812

Tan, J. C., Smith, B. D., \& O'Shea, B. W. 2010, in AIP Conf. Ser. 1294, The

First Stars and Galaxies: Challenges for The Next Decade, ed. D. J. Whalen, V. Bromm, \& N. Yoshida (Melville, NY: AIP), 34

Tanaka, T., \& Haiman, Z. 2009, ApJ, 696, 1798

Tornatore, L., Ferrara, A., \& Schneider, R. 2007, MNRAS, 382, 945

Trenti, M., Stiavelli, M., \& Michael Shull, J. 2009, ApJ, 700, 1672

Turk, M. J., Abel, T., \& O’Shea, B. 2009, Science, 325, 601

van Wassenhove, S., Volonteri, M., Walker, M. G., \& Gair, J. R. 2010, MNRAS, 408, 1139

Volonteri, M., Haardt, F., \& Gültekin, K. 2008a, MNRAS, 384, 1387

Volonteri, M., Haardt, F., \& Madau, P. 2003, ApJ, 582, 559

Volonteri, M., Lodato, G., \& Natarajan, P. 2008b, MNRAS, 383, 1079

Wadsley, J. W., Stadel, J., \& Quinn, T. 2004, New Astron., 9, 137

Wehner, E. H., \& Harris, W. E. 2006, ApJ, 644, L17

Wise, J. H., Turk, M. J., \& Abel, T. 2008, ApJ, 682, 745

Yan, R., Ho, L. C., Newman, J. A., et al. 2011, ApJ, 728, 38 